\title{
THE ABSORPTION-LINE QSOS
}

\author{
C. R. LYNDS
}

Kitt Peak National Observatory, Tucson, Ariz., U.S.A.

\begin{abstract}
Many features of the distribution of emission and absorption line redshifts of QSO spectra and of the distribution of the differences between emission and absorption line redshifts may be due to observational selection. However, it seems significant that all six of the absorption line objects of largest redshift have multiple absorption systems. Observations seem to favor more strongly the hypothesis that the multiple absorption line systems are due to clouds associated with the QSO rather than in the intervening space. The multiple absorption line systems of the QSOs PKS 0237-23, Ton 1530 and $4 C 05.34$ are discussed. The persistence of the Lyman- $\alpha$ absorption feature and identification of multiple-absorption line systems strongly suggests that unidentified absorption lines shortward of the Lyman- $\alpha$ emission also arise from the Lyman- $\alpha$ transition.
\end{abstract}

I have collected together all of the information available to me on the spectroscopic characteristics of QSOs relevant to the occurrence of absorption lines. Figure 1 shows the emission-line redshift distribution for 236 out of a sample of 237 objects. The QSO omitted, although confirmed to be extragalactic, is only known to have absorption lines. The spectra of 60 objects in Figure 1 show absorption lines with sufficient certainty that it is possible to make proposals for their identification and redshift. These objects are indicated by the total cross-hatched area in Figure 1. A dozen or more additional objects are suspected of having absorption lines. The spectra of many QSOs appear to indicate the presence of more than one absorbing cloud. Such objects are indicated in Figure 1 by additional cross-hatching.

Superficially, the redshift distribution for all QSOs shows a steep increase out to a redshift of approximately 0.4 and a steady decline until what appears to be a statistically significant steep decline at a redshift of about 2.1 . If we were dealing with a sample that was complete over the entire range of redshift, the numbers would increase steadily - sharply at first but slowing somewhat toward larger redshift. The initial steep rise in the observed distribution may indicate that the sample is to some extent representative out to a redshift of about 0.3 , but the subsequent decline illustrates the enormous incompleteness in the sample. The incompleteness at a redshift of 2.1 is so severe that physical interpretations of the steep decline should probably await a more complete understanding of the factors that may influence observational selection.

The apparent redshift distribution for the absorption-line objects is somewhat different from that for all objects taken together. There is no evidence for a steady decline with redshift, and there is what appears to be a peak at a redshift of 2.0. The detection of absorption lines in objects depends strongly on the MgII resonance doublet ( $\lambda 2798)$ out to a redshift of about 1.1. The fact that this feature cannot be observed for redshifts less than about 0.2 explains why there are no absorption-line objects with redshifts less than this value. For redshifts larger than 1.1 the CIV resonance doublet $(\lambda 1549)$ plays an increasingly important role in the detection of 
absorption, and there are several reasons to expect that this feature is more persistent - that is, can be more easily detected when small quantities of material are involved than the MgII lines. This effect may explain why the redshift distribution does not show the decline that is present for all of the QSOs taken together. It is also important

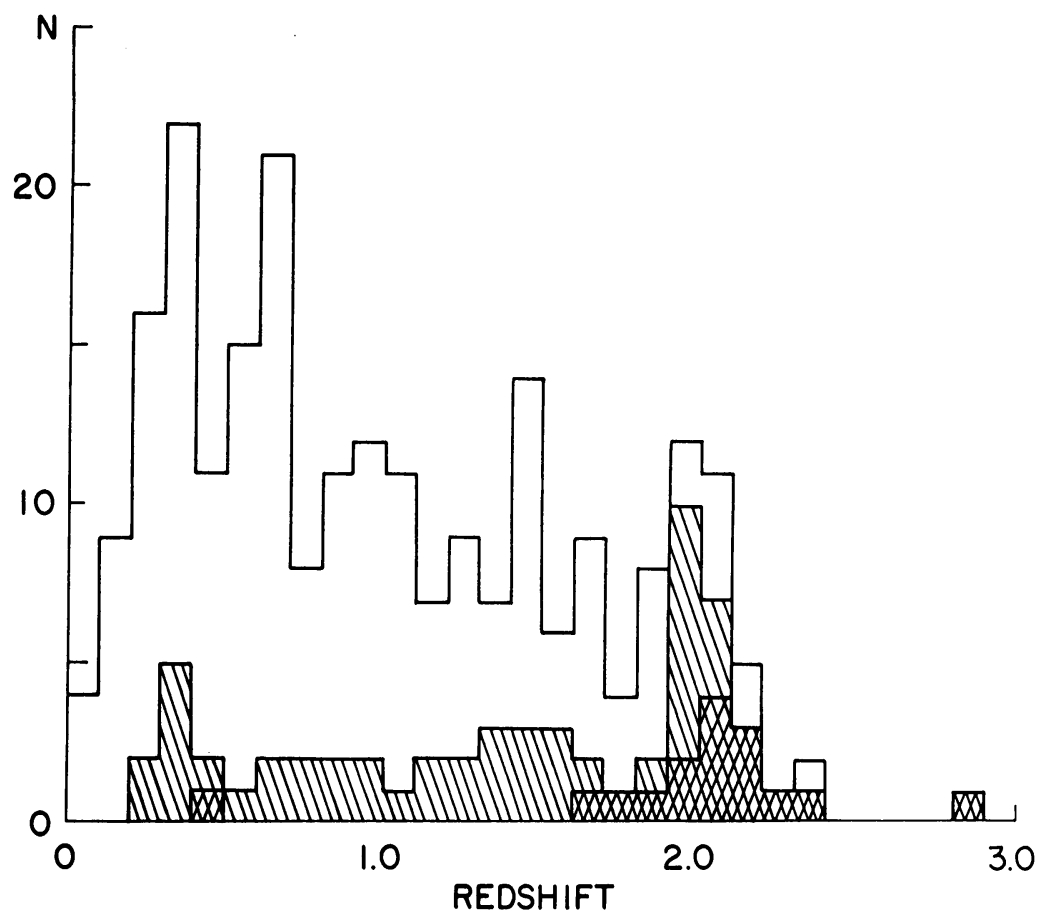

Fig. 1. The distribution of emission-line redshift for 236 QSOs. The entire cross-hatched area represents all absorption-line QSOs, and the area having additional cross-hatching denotes those objects that have multiple absorption systems.

to note that there is a considerable body of evidence indicating that Lyman- $\alpha$ of hydrogen is usually the most persistent feature arising in the clouds responsible for the absorption spectra. This, together with the fact that the region of Lyman- $\alpha$ cannot be really well observed for redshifts smaller than about 1.9, may explain, at least in part, the prevalence of absorption lines for objects having redshifts near 2.0. However, it should be remembered that many of the large-redshift objects not noted as having absorption lines are very faint and difficult to observe sufficiently well to rule out the presence of absorption lines comparable in strength to those found in the other sources.

The redshift distribution of objects having multiple absorption redshifts may well be influenced by the factors just mentioned, but the fact that all six of the absorptionline objects of largest redshift have multiple absorption systems appears to me to be significant. Furthermore, the three objects with redshifts greater than 2.2 appear to have many more than one or two absorption redshift systems. 
It is instructive to look at the distribution of redshift residuals, $z_{\mathrm{abs}}-z_{\mathrm{cm}}$ for the absorption systems in all of the absorption-line QSOs. This distribution is shown in the lower part of Figure 2. (Actually, certain absorption systems characteristic of the largest-redshifts objects have been excluded because they would have dominated the distribution. These absorption systems will be discussed later.) There is seen to be a

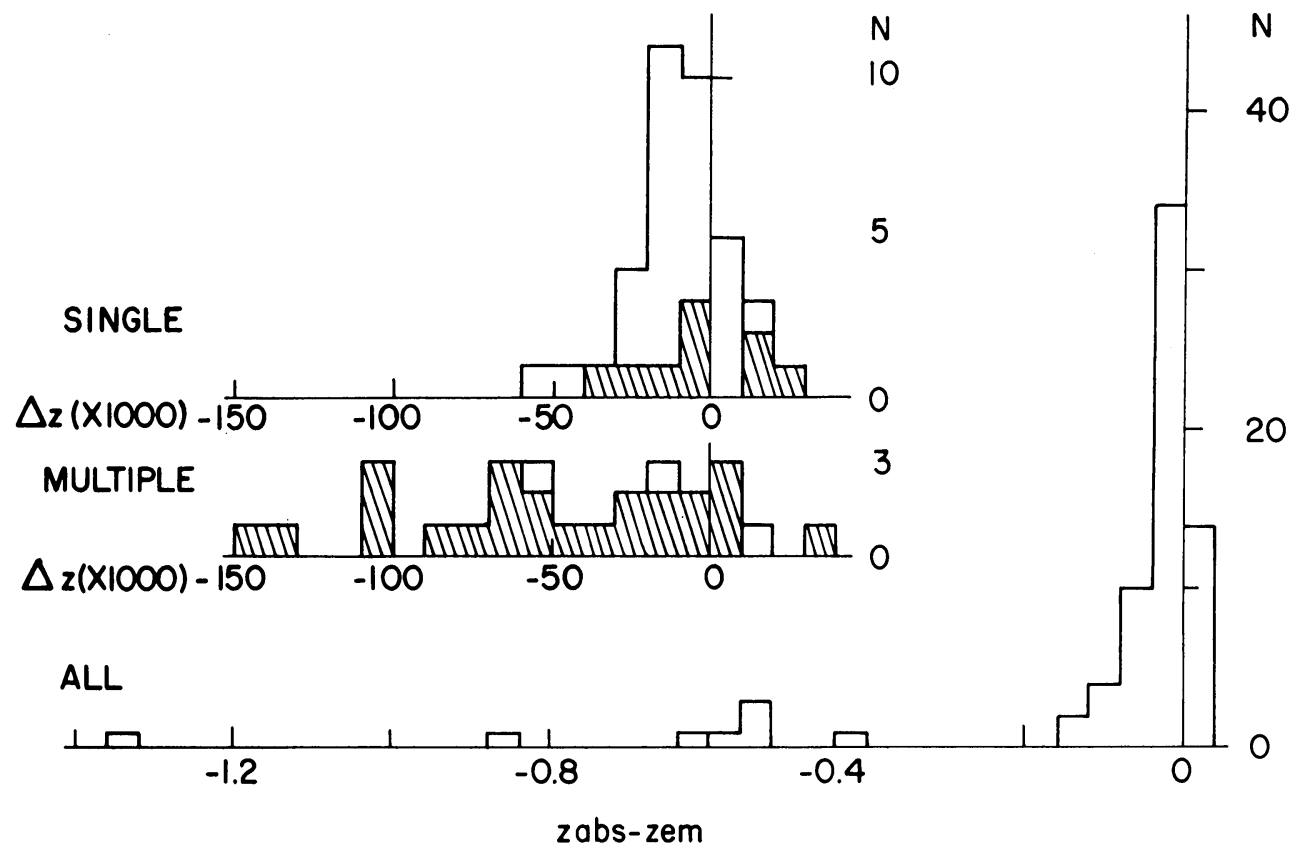

Fig. 2. The distribution of absorption redshift residuals, $z_{\mathrm{abs}}-z_{\mathrm{em}}$, for all absorption-line QSOs and for single-system and multiple-system QSOs separately. The cross-hatched area indicates those objects having emission-line redshifts greater than 1.8 .

strong tendency for the absorption-line redshifts to be near the emission-line value or, more exactly, 0.02 less than the emission-line value. The distribution is quite compact and the largest positive residual is 0.031 . There are, however, eight systems showing very large negative residuals, and these eight systems are present in the spectra of only four objects, one of which accounts for five of the systems. These four objects all have more than one absorption system and have at least one system with a redshift near the emission-line value.

From the appearance of the distribution of residuals in Figure 2, one might speculate that there are two classes of absorption-line QSO: those with single absorption systems having redshifts always near the emission-line value and those with multiple systems distributed over a much larger range of redshift. The two upper parts of Figure 2 show, on an expanded scale, the distribution of residuals for each of the two classes of QSO. It is seen that there appears to be a major difference between the two distributions, with the single-system QSOs showing a much stronger 
tendency for small residuals than do the multiple-system objects. However, the bulk of the single-system objects have redshifts smaller than about 1.8 and depend on transitions (MgII $\lambda 2798$ and CIV $\lambda$ 1549) presumably less effective than Lyman- $\alpha$ in revealing the presence of multiple systems. Therefore, it is more germane to the question to look at the distribution of residuals for only those objects having redshifts greater than 1.8. These objects are indicated by cross-hatching in the illustration. It is seen that there remains some indication that single absorption systems tend to have small residuals, but the significance of the effect is placed somewhat in doubt by the fact that only two, or at most four, of the nine single-system objects can be considered to have been intensively studied.

The distribution of redshifts for the absorption systems - in both absolute value and relative to the emission-line redshifts of the objects - is of particular importance to the question of the location and origin of the media responsible for the absorption lines. For example, the strong tendency for QSOs to show small absorption-redshift residuals, for at least the more prominent absorption systems, strongly implies that the absorbing media are located in the immediate vicinity of the objects. On the other hand, those QSOs having multiple absorption systems tend to fit more naturally into the notion that the absorbing material is distributed in discrete clouds throughout the intervening space between such objects and the observer and that the redshifts are cosmological in origin. There is no a priori reason why both situations cannot obtain, with the observed absorption lines having both an intrinsic and an extrinsic origin, as they do in the case of galactic stars. Finally, there is the possibility that all of the observed absorbing material is uniquely associated with the particular QSOs in which they are observed and that the clouds showing very large redshift residuals actually have their origin and their large differential motions as a consequence of an energetic expulsion of material from the QSO. This latter interpretation has some difficulty, not necessarily insurmountable, in accounting for the extreme narrowness of the observed absorption lines in comparison with the inferred expulsion velocity.

In connection with the possibility that some of the absorbing clouds, especially those showing large redshift residuals, are cosmological in nature and not associated with the particular objects in which they are observed, it is important to realize that there should be a correspondence between the space density of such clouds and the probability of finding clouds at particular redshifts in the spectrum of a QSO. Thus, if the clouds were uniformly distributed throughout the Universe, there should be observed no statistically significant deviation from uniformity in the observed redshift distribution of the clouds. The indication from the present sample of multiple redshift QSOs is that the distribution of redshift is more-or-less uniform with a probably significant tendency for there to be more absorbing clouds located at large redshift.

This interpretation is, however, confronted with some evidence of a striking contradiction. If the absorbing clouds are presumed to be cosmological in distribution, any of the QSOs with emission-line redshifts larger than a particular value should serve equally well - observational factors being equal - as probes in revealing the presence 
of the clouds. Therefore, it is noteworthy that, of the 44 objects having redshifts greater than 1.7 and thus capable of revealing the presence of clouds of smaller redshift, only three objects do reveal such clouds. These three objects have multiple absorption systems and show seven clouds, all having redshifts considerably less than the emission-line redshifts of the objects. This would seem to represent a rather large statistical fluctuation within the framework of the hypothesis that such clouds are cosmological in distribution. In fact, if the case were any stronger, one might be compelled to conclude that observed clouds are in every case peculiarly associated with the particular objects in which they are observed. As a note of caution, however, it must be confessed that the three QSOs in question have been more intensively studied than almost all of the other QSOs.

The distilled characteristics of absorption-line QSOs that have been presented indicate, in spite of the many necessary observational reservations, that the attribute of absorption lines shows a variation of degree as a function of redshift; absorption lines become an increasingly dominant characteristic as one passes to objects of very large redshift. This impression is considerably strengthened by a familiarity with the nature of the original observational material. As I have had the privilege of working with a considerable amount of such material, it occurs to me that it may be of interest to present in the time remaining some of this along with my views as to its interpretation. In particular, the entire discussion will pertain to a hypothesis used as a basis for a likely interpretation of the multitude of 'unidentified' absorption lines found in objects of large redshift.

The immense complexity of the absorption spectra of objects of large redshift requires an appreciation of the formidable observational problem and the importance of spectral resolution. Some of the effects of improving spectral resolution are illusstrated in Figure 3, where a portion of the very complex spectrum of Ton 1530 is reproduced from six spectrograms ranging in resolving power from $10 \AA$ to $1 \AA$. (As a technical note, all spectrograms except the one of highest dispersion have been

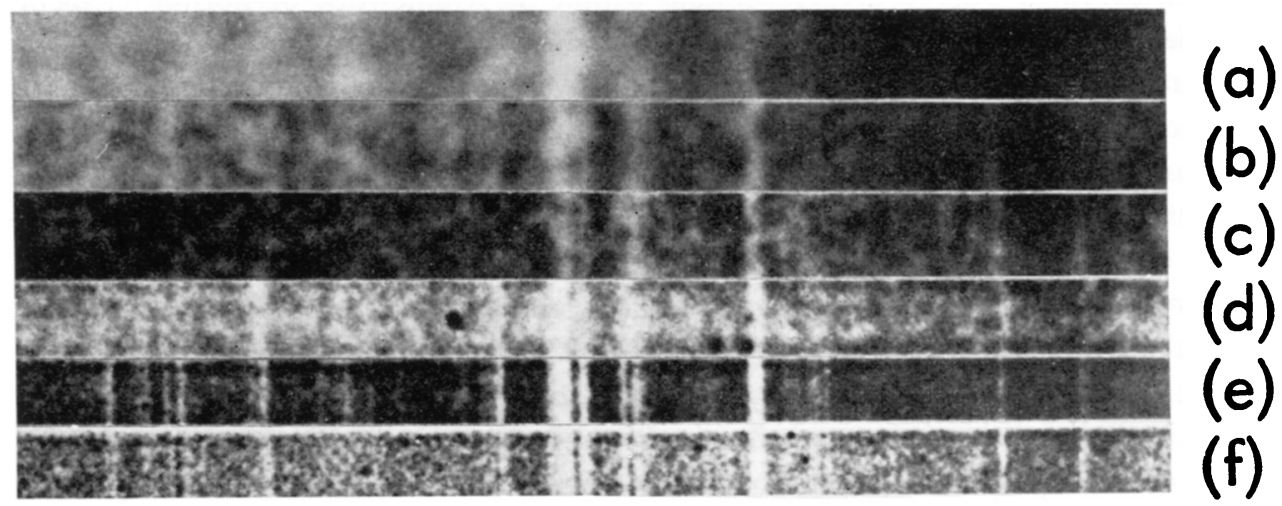

Fig. 3. The effects of spectral resolution as shown by six spectrograms of Ton 1530 ranging in resolution from $10 \AA$ for spectrum $(a)$ to $1 \AA$ for spectrum $(f)$. 
photographically 'folded' so that the information content per resolution element is roughly the same for all spectrograms reproduced. This exacting prccess was accomplished by Mr Hanna of the Kitt Peak National Observatory photographic laboratory.) It is seen that high resolution is not only important for revealing weak and narrow lines and resolving closely-positioned lines, but that it is essential for clarifying the true character of complex blends involving both weak and strong lines. The narrowest lines in spectrum $(f)$ of Figure 3 are saturated and unresolved with a width of the order of $1 \AA$ - or $0.3 \AA$ in the rest frame of this particular source. Because of the faintness of most QSOs and the consequent fact that a successful effort at the highest possible dispersion usually requires nearly perfect observing conditions, we seldom have the luxury of spectrograms of the quality of spectrum $(f)$ and must in many cases be satisfied with spectrograms resembling spectrum $(a)$.

The difficulties of obtaining adequate spectral resolution, when compounded with the presence of multiple redshifts and the possibility of spectrum variations, tend to place added importance on physical reasonableness as a consideration in making line identifications. In my opinion it is also prudent to preserve a certain amount of 'continuity' in the approach to line identification in new and especially in difficult objects; the interpretations that have been found to be successful and wellreasoned in previously studied objects should form much of the basis for the analysis.

One of the first absorption-line QSOs to be discovered and perhaps the one that can inspire the most confidence in the subsequent analyses of other objects is $3 \mathrm{C} 191$. The spectrum of this object contains broad emission lines that can with considerable confidence be identified with Lyman- $\alpha$ of hydrogen, the resonance transitions of SiIV ( $\lambda 1397)$ and CIV ( $\lambda 1549)$, the 'Balmer- $\alpha$ ' transition in HeII ( $\lambda 1640)$, and the lowest inter-system transition in CIII ( $\lambda 1909)$. A portion of the confidence in these identifications stems from the early demonstration by Schmidt (1965) of continuity of spectroscopic characteristics in going from the firmly-established interpretations of small-redshift objects to the large redshift (2.012) of 3C 9. Furthermore, the line identifications in 3C 191 are reasonable within the framework of normal element abundances and self-consistent excitation conditions.

If any additional support for the validity of the emission-line identifications was required, it was forthcoming in the spectrum of 3C 191. This evidence takes the form of numerous narrow absorption lines that may be conclusively identified with groundstate transitions in hydrogen, SiII, SiII, SiIv, Nv, CiI, and Civ at a redshift very nearly equal to the emission-line value. A previously published spectrogram of $3 \mathrm{C} 191$ is reproduced in Figure 4. Notice particularly that the proximity between emission and absorption lines is perfectly natural within the framework of an implied physical and geometrical relationship between emitting and absorbing material. The evidence for such a relationship is strengthened in the present case by the fact that some of the observed absorption lines arise from excited fine-structure levels within the ground states, suggesting that the emitting body may provide the necessary excitation. Among the many absorption-line systems in subsequently studied objects, I know of none 


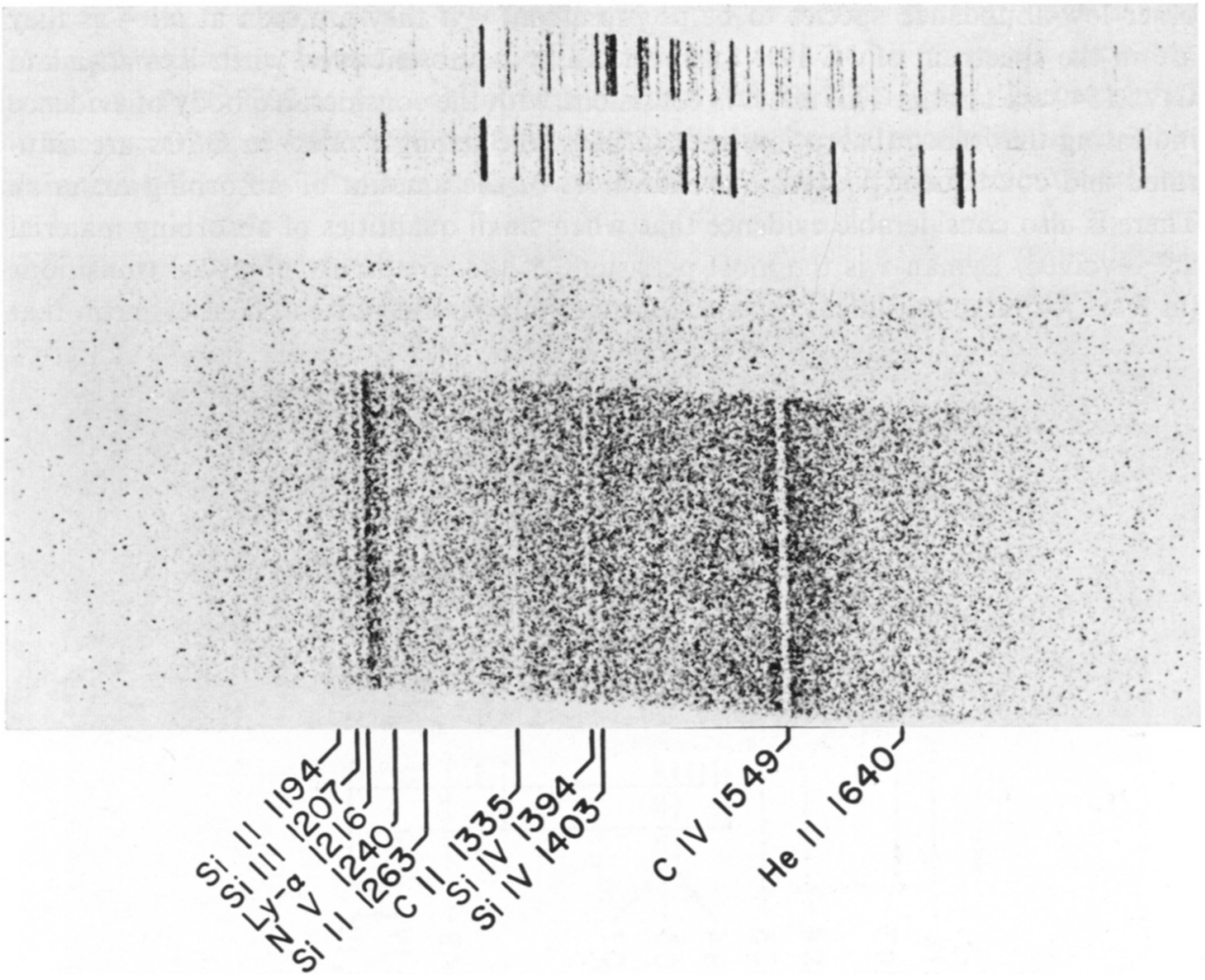

Fig. 4. A spectrogram showing the well-developed absorption spectrum of 3C 191.

that gives clear evidence for population of excited fine-structure levels whenever the absorption-line redshift differs greatly from the emission-line value.

The proximity between absorption and emission lines or, more generally, an apparently special kinematic relationship between absorbing and emitting material may form a tentative basis for identification of an absorption feature, even when there is only one. Thus, in one of the first absorption-line QSOs discovered, Sandage (1965) was quite justified in suggesting that the absorption line occurring in the middle of the CIV $\lambda 1549$ emission in BSO 1 was due to the same transition in absorption. This point is especially relevant in view of the fact that a large fraction of absorptionline QSOs display only one known absorption line. In essentially all cases such a line is found to be in or near an emission line due to Lyman- $\alpha$, CIV $\lambda 1549$, or MgII 22798. The provisional assumption that the identification of the absorption line is the same as the associated emission line is very likely to be correct, for in several cases I have checked by obtaining improved observations, only one (PHL 938) was found to be erroneous.

Because the spectrum of 3C 191 is exceptional and can be misleading, it should be mentioned that in other QSOs one seldom finds the lines of the ions of silicon and 
other low-abundance species to be as prominent - if they are seen at all - as they are in the spectrum of $3 \mathrm{C} 191$. Such lines may be absent even when Lyman- $\alpha$ and CIV $\lambda 1549$ are strong. This effect is consistent with the considerable body of evidence indicating that most absorption lines (at least the stronger ones) in QSOs are saturated and consequently insensitive indicators of the amount of absorbing material. There is also considerable evidence that when small quantities of absorbing material are involved, Lyman- $\alpha$ is the most persistent of the commonly observed transitions (at least for large-redshift objects). However, this should not be taken to mean that

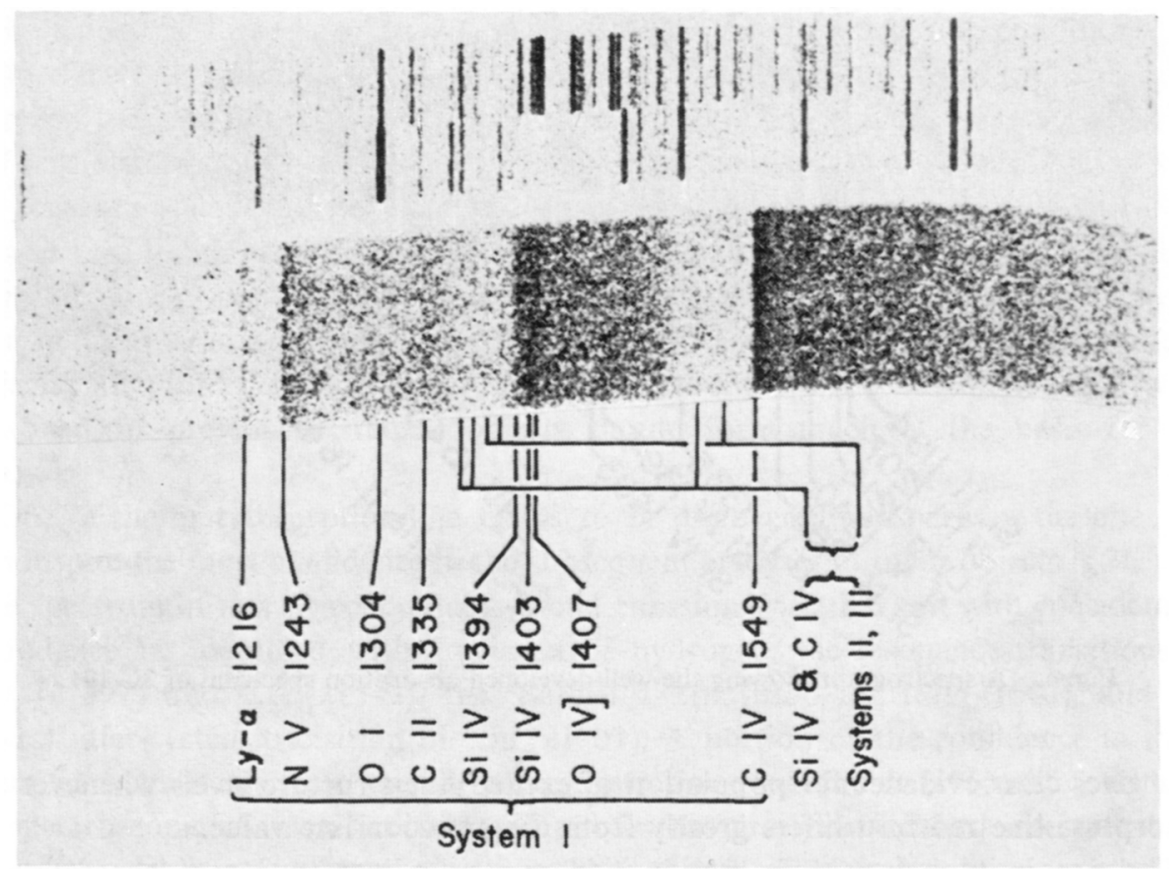

Fig. 5. A spectrogram showing the broad absorption bands in PHL 5200. Systems II and III refer to structure that appears superficially as emission.

there is no evidence for small variations of element abundances or level of excitation in passing from one object to another.

The probable relevance of a special relationship between the redshift distributions for absorbing and emitting material is further illustrated by the spectrum of PHL 5200 illustrated in Figure 5. Emission lines, unquestionably due to the resonance transitions of hydrogen, $\mathrm{N} \mathrm{v}, \mathrm{Si}$ IV, and $\mathrm{C} \mathrm{IV}$, are bordered on their violet sides by extremely broad absorption bands. Not only does the identification of the absorption and emission features with the same transitions follow naturally from the obvious kinematic relationship, but the interpretation is also supported by the coherence of the structure within the SiIV band with that within the CIV band. Implicit in this interpretation of the spectrum of PHL 5200 is the distinct possibility of a generic con- 
nection between objects of this type and the multiple-absorption-redshift QSOs. Thus, a simple reduction in the amount of absorption at all redshifts would probably transform PHL 5200 into an object with relatively distinct absorption-line redshifts.

The spectrum of PHL 5200 tends to lend credibility to the possibility of multiple absorption-line redshifts, but it did not completely foretell the extreme situation

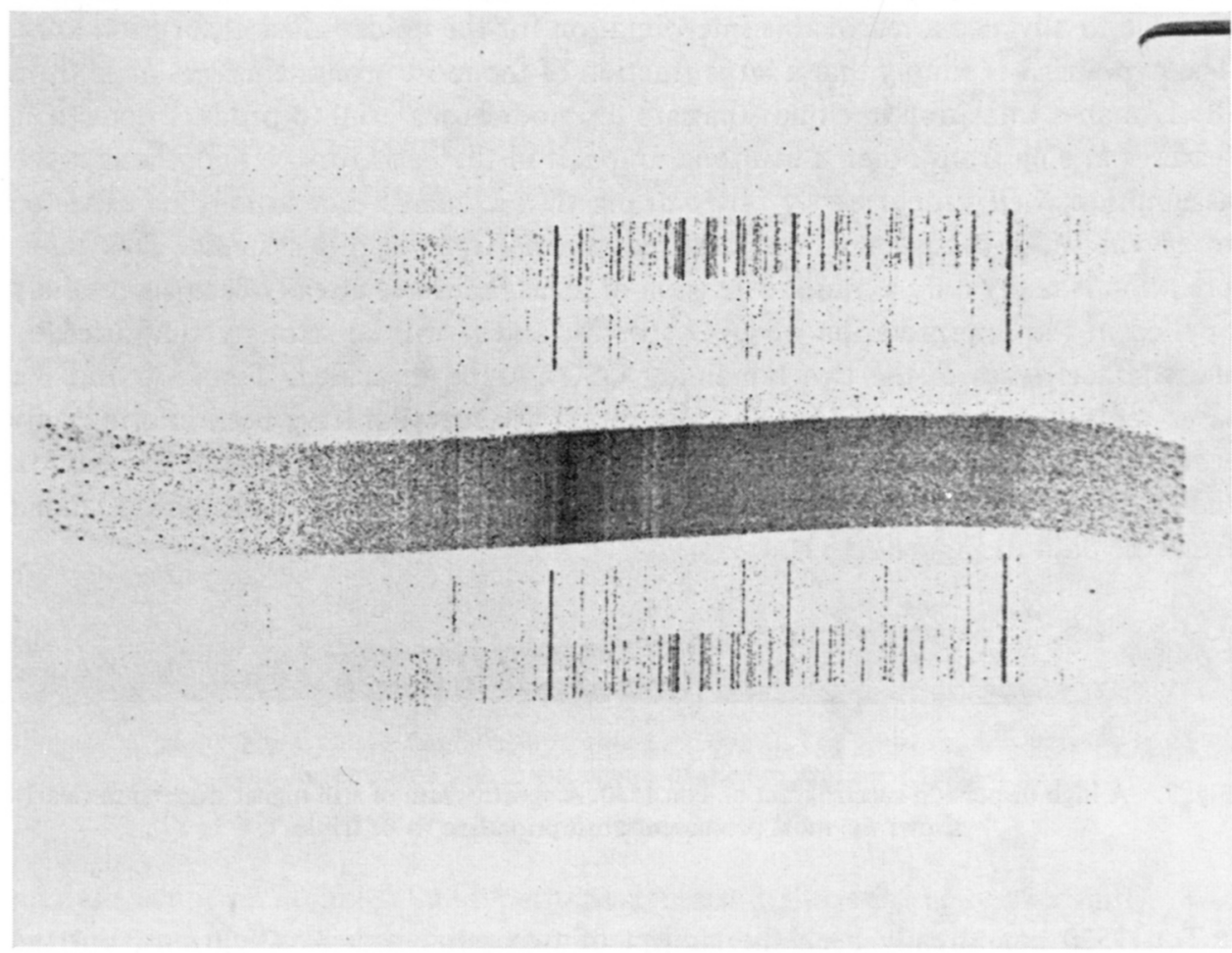

Fig. 6. A relatively low dispersion spectrogram of PKS 0237-23. Many of the absorption features are shown to have fine structure and many additional weak lines are revealed by higher dispersion spectrograms.

found in PKS 0237-23. A low-dispersion spectrogram of this object reproduced in Figure 6 shows the strong and very broad Lyman- $\alpha$ emission line, a less prominent Civ $\lambda 1549$ emission line, and numerous absorption features. The application of higher dispersion reveals the presence of many additional lines. The initial attempts to account for the lines in one or two redshift systems were not particularly successful in explaining the more prominent features. However, the analysis has now reached the state where several investigators would probably agree to the presence of approximately six redshift systems. These include a system with a redshift near the emissionline value of 2.22 and several systems with considerably smaller redshifts, the smallest of which is 1.364 and is well-established by the presence of several prominent and important transitions. 
These absorption systems were essentially all that could be established by what might simply be described as a 'pattern-recognition' method, in spite of the fact that many lines were left without identifications. However, almost all of the prominent unidentified lines occur on the short-wavelength side of the Lyman- $\alpha$ emission line. This fact must be recognized as representing a semblance of order and regularity in the data and, as such, can form the basis of a hypothesis by means of which it is possible to advance a reasonable interpretation for the unidentified absorption lines. The hypothesis is simply that a large fraction of the most prominent lines arise from the Lyman- $\alpha$ transition in clouds that are too poor in material to produce detectable features arising from other transitions. Implicit in the basis for the hypothesis is the assumption, well supported by observation, that Lyman- $\alpha$ can usually be expected to be the most persistent absorption feature in large-redshift systems. This interpretation is really only a minor extension of ideas that have already been successfully applied in the interpretation of QSO spectra, and it will be strongly supported by the characteristics of the two remaining QSOs to be discussed, Ton 1530 and the large-redshift object $4 \mathrm{C} 05.34$. (The absorption systems that have been inferred only from the prevalence of absorption lines near and shortward of Lyman- $\alpha$ emission in the large-redshift objects are the ones that were referred to as having been excluded from the data in Figure 2.)

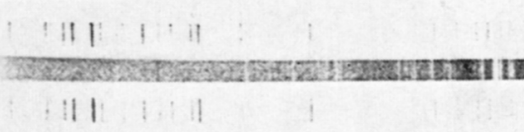

Fig. 7. A high dispersion spectrogram of Ton 1530. A spectrogram of still higher dispersion clearly shows the most prominent absorption line to be triple.

Ton 1530 has already been the subject of two published investigations, but the present discussion is based on new observational material. One of several recent spectrograms of the object is reproduced in Figure 7. The Lyman- $\alpha$ emission line in the center of the spectrum is seen to be flanked on the violet side by numerous narrow absorption lines. Only a few absorption lines have been found longward of Lyman- $\alpha$ (out to about $5000 \AA$ ). By the technique of 'pattern-recognition' it has been possible to establish the presence of six or seven absorption-line redshifts (two of the systems have nearly the same redshift), one of which is much smaller than the emission-line value -1.486 , as compared with 2.046 . These redshifts serve to identify nearly all detectable absorption lines longward of Lyman- $\alpha$ but leave most of the lines shortward of Lyman- $\alpha$ without identifications. The distribution of the unidentified lines represents the same type of regularity as was found in PKS 0237-23 and indicates that a large fraction of the lines may reasonably be interpreted as arising from Lyman- $\alpha$.

The QSO 4C 05.34 shows an extreme version of the same type of absorption spectrum as PKS 0237-23 and Ton 1530 (as well as several other sources for which the 


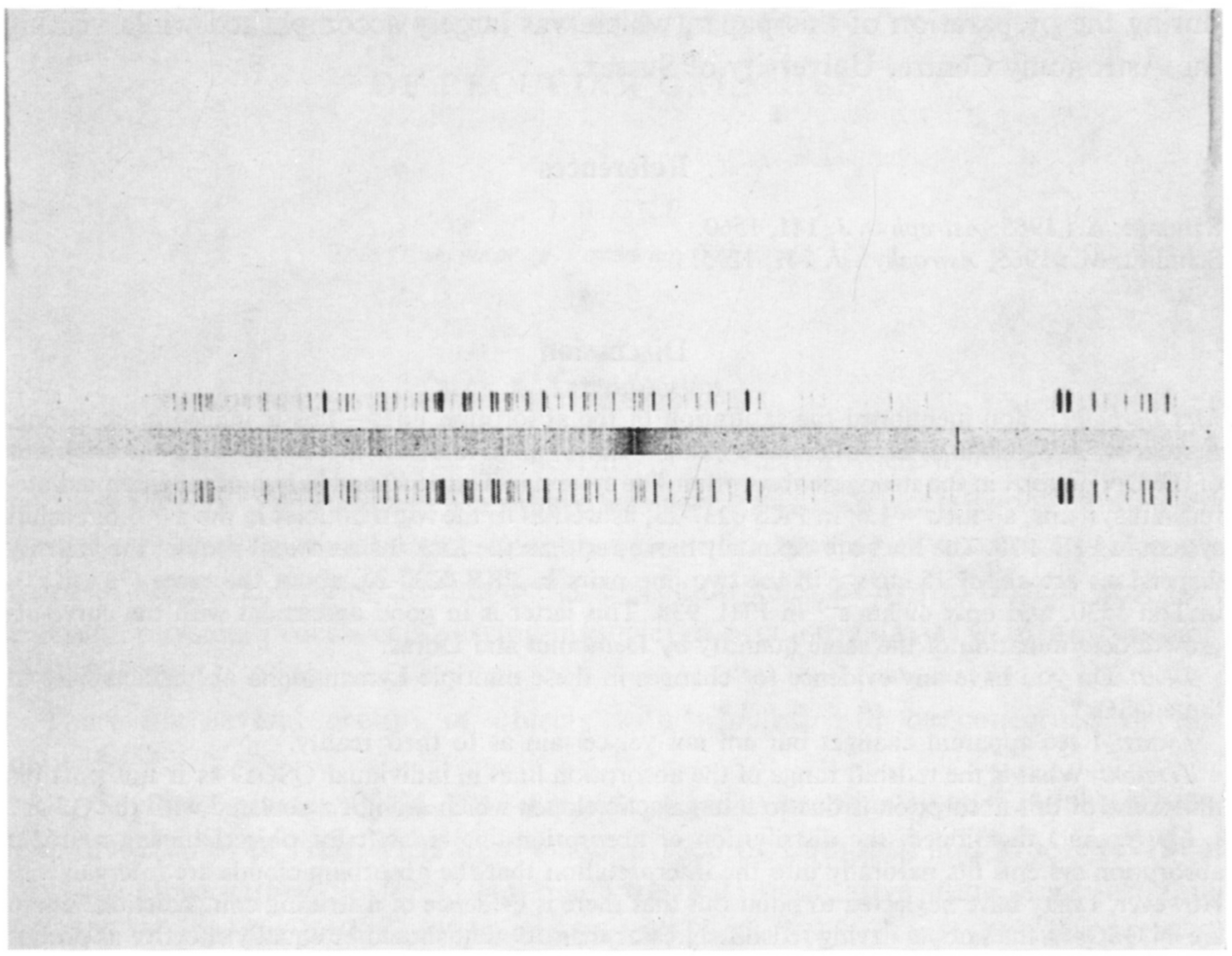

Fig. 8. A spectrogram of the large-redshift object $4 \mathrm{C}$ 05.34. The emission-line redshift is 2.877 , and the emission line in the center of the spectrum is Lyman- $\alpha$.

analysis is not yet complete) and, perhaps significantly, has the largest redshift, 2.877 , known for a QSO. A recent spectrogram of this object is reproduced in Figure 8. This and several other spectrograms show few prominent absorption lines longward of the Lyman- $\alpha$ emission, located in the center of the spectrogram, but a very large number of lines shortward of Lyman- $\alpha$. Approximately 100 reasonably definite absorption lines have been measured in this object. By the procedure of 'pattern-recognition' at least five absorption-line redshifts have been established; the largest is 2.875 , near the emission-line value, and the smallest is 2.473 . These systems account for all of the prominent lines longward of Lyman- $\alpha$ but only a small fraction of those shortward of Lyman- $\alpha$. The very special arrangement of the unidentified absorption lines with respect to emission in a transition having the astrophysical importance of Lyman- $\alpha$ leaves little doubt in my mind that the correct interpretation of the lines is that they also arise from Lyman- $\alpha$.

\section{Acknowledgements}

I wish to acknowledge helpful discussions with T. D. Kinman and W. H. McCrea 
during the preparation of this paper, which was largely accomplished while visiting the Astronomy Centre, University of Sussex.

\section{References}

Sandage, A.: 1965, Astrophys. J. 141, 1560.

Schmidt, M.: 1965, Astrophys. J. 141, 1275.

\section{Discussion}

Mrs Burbidge: You mentioned the saturation of the absorption lines - this is indeed quite strong. A student, Y-W. Chan at La Jolla, has applied the Strömgren line-pair method to Lick spectrograms of the Civ doublet in the strongest absorption-line system in Ton 1530 and in two of the intermediateredshift systems, about $z=1.6$, in PKS 0237-23, as well as to the MgII doublet in the $z=0.6$ redshift system in PHL 938. The lines are definitely narrower than the Lick instrumental profile; the velocity dispersions are about $75 \mathrm{~km} \mathrm{~s}^{-1}$ in the two line pairs in PKS 0237-23, about the same $\left(73 \mathrm{~km} \mathrm{~s}^{-1}\right)$ in Ton 1530, and only $49 \mathrm{~km} \mathrm{~s}^{-1}$ in PHL 938 . This latter is in good agreement with the curve-ofgrowth determination of the same quantity by Demoulin and Doras.

Dent: Do you have any evidence for changes in these multiple Lyman-alpha absorption lines in these QSOs?

Lynds: I see apparent changes but am not yet certain as to their reality.

Terzian: What is the redshift range of the absorption lines in individual QSOs? Is it not possible that some of this absorption is due to intergalactic clouds which are not associated with the QSOs?

Lynds: As I mentioned, the distribution of absorption-line redshift for objects having multiple absorption systems fits naturally into the interpretation that the absorbing clouds are intergalactic. However, I may have neglected to point out that there is evidence of a striking contradiction. There are 44 QSOs in the sample having redshifts greater than 1.7 that should be equally effective as probes in revealing the presence of absorption by intergalactic clouds with redshifts less than 1.7. In fact, however, only three objects do so, and these three objects have multiple absorption systems with at least one absorption system near the emission-line redshift which is 1.955 in the object with the smallest emission redshift. Furthermore, these three objects show seven absorption redshifts less than 1.7 , five of which are revealed by only one of the three objects. This would seem to be a rather large statistical fluctuation within the framework of a hypothesis that the absorbing clouds are cosmological in distribution. It must be confessed that the three QSOs in question are perhaps the most intensively studied of all QSOs. On the other hand, this reservation does not appear to be particularly strong and the weight of evidence may well compel us to conclude that absorbing clouds are in every case peculiarly associated with the particular objects in which they are observed.

Mrs Rubin: What can you say about the absorption line velocity systems which are more positive than the emission line velocity systems in a single object?

Lynds: The largest positive redshift residual in my sample is 0.031 , corresponding to about $3000 \mathrm{~km} \mathrm{~s}^{-1}$ for redshifts near 2.0. This is comfortably within acceptable velocity dispersions for the corresponding cosmological epoch.

Ozernoy: If the absorbing gas is in the QSOs themselves, what is your estimate of the total mass of gas clouds?

Lynds: I have made no estimate.

Roeder: I have just finished using the statistical test of Bahcall and Peebles for the randomness of the absorption-line systems, applied in several different General Relativity models of the universe. If one uses all absorption-line systems, one finds that they are strongly non-random. If one uses only the three or four objects with narrow absorption lines, one finds again that the absorption redshift systems seem to be non-random, although of course this conclusion is not extremely strong because of the small number of objects. 